\title{
Sustainable antenatal care services in an urban Indigenous community: the Townsville experience
}

\author{
Kathryn S Panaretto, Melvina R Mitchell, Lynette Anderson, Sarah L Larkins, Vivienne Manessis, Petra G Buettner and David Watson
}

$\mathrm{R}$ ates of preterm births, low birthweight and perinatal mortality in the Australian Indigenous population have remained unacceptably high over the past decade, reflecting failure of policy and health care delivery. ${ }^{1,2}$ Improving perinatal outcomes is heavily dependent on improving the social determinants of health, ${ }^{3}$ but the health sector has an opportunity to intervene and influence outcomes by providing good antenatal care. ${ }^{4}$

Since late 1999, health service providers in Townsville, North Queensland, have worked closely with the Indigenous community to improve antenatal services. This collaboration has resulted in an integrated model of shared antenatal care, the Mums and Babies program, delivered from the Townsville Aboriginal and Islanders Health Service (TAIHS). Initial evaluation of this program showed improved access and a reduction in preterm births, but unchanged perinatal mortality. ${ }^{5}$

Here, we report the results of sustaining the Mums and Babies program for 6 years. We aimed to assess the continued impact of the program by evaluating access to care, quality of service delivery, and effects on perinatal outcome.

Townsville has an Indigenous population of 7675 (5.0\% of the population), and at the last census there were 16875 Indigenous people $(5.2 \%)$ in the Townsville region (70\% Aboriginal and 30\% Torres Strait Islander descent). ${ }^{6}$ TAIHS is an Aboriginal medical service with a 10-member elected board and over 100 staff members. Most Indigenous births in Townsville occur at the Townsville Hospital, the tertiary referral centre for North Queensland.

\section{METHODS}

\section{Study design}

The study is a before-and-after evaluation of a quality improvement intervention at TAIHS that targeted all pregnant women attending for shared antenatal care. All women who made at least one antenatal visit with the Mums and Babies program, and gave birth to an Indigenous infant between 1 January 2000 and 31 December 2005, formed the intervention group (MB group).

\section{ABSTRACT}

Objective: To evaluate the impact of a sustained, community-based collaborative approach to antenatal care services for Indigenous women.

Design: Prospective quality improvement intervention, the Mums and Babies program, in a cohort of women attending Townsville Aboriginal and Islanders Health Service, 1 January 2000 - 31 December 2005 (MB group), compared with a historical control group (PreMB group), 1 January 1998 - 30 June 1999.

Main outcome measures: Proportion of women having inadequate antenatal care and screening; perinatal indicators.

Results: The number of antenatal visits per pregnancy increased from three (interquartile range [IQR], two to six) in the PreMB group to six (IQR, four to ten) in the MB group $(P<0.001)$. There were significant improvements in care planning, completion of cycle-of-care, and antenatal education activities throughout the study period. About $90 \%$ of all women attending for antenatal care were screened for sexually transmitted diseases, $89 \%$ had measurement of haemoglobin level, and serological tests for hepatitis B and syphilis (minimum antenatal screening). There was increased attendance for dating and morphology scans. In the MB group compared with the PreMB group, there was a significant reduction in perinatal mortality (14 v 60 per 1000 births; $P=0.014$ ). Conclusion: Sustained access to a community-based, integrated, shared antenatal service has improved perinatal outcomes among Indigenous women in Townsville.

MJA 2007; 187: 18-22

To assess the impact of the intervention, we have used a historical control group of women (the PreMB group) who attended TAIHS for antenatal care and gave birth between 1 January 1998 and 30 June 1999.

Intervention: an integrated model of antenatal shared care

The Mums and Babies program, which has been described previously, ${ }^{5}$ commenced on 1 January 2000, integrating three formerly independent providers of antenatal care: TAIHS, and Queensland Health services the Community Child Health Service and the Institute of Women's and Children's Health at Townsville Hospital. The program has a young-family focus (not the traditional antenatal-postnatal care model), and is open to all pregnant women and families with children under the age of 8 years. The program has evolved within a quality improvement framework, including annual clinical audit, client survey and regular program refinement.

\section{Management}

Standard Townsville Hospital antenatal shared-care protocols, based on those of the Royal Australian and New Zealand College of Obstetricians and Gynaecologists, were used, with additional infection screening: self-administered screening for sexually transmitted infections and group B streptococcal infection, and a repeat urine culture. Patients with high-risk or complicated pregnancies were referred to Townsville Hospital at the discretion of the attending medical staff.

\section{Ethics approval}

The program and evaluation have the full support of the community-elected Board of TAIHS. The ethics committee of the Townsville District Health Service approved the evaluation. All participating women were aware of data collection activities at TAIHS, and provided verbal consent to participate in evaluation activities during the first visit. No women refused to participate.

\section{Ethnicity}

Staff asked all women presenting for antenatal care about their ethnicity. A baby was identified as Indigenous if either parent identified as such; Indigenous infants comprised $94 \%$ of all births to women receiving antenatal care at TAIHS during the study period. 


\section{Data collection}

Data on the MB group were collected prospectively. A comprehensive, confidential record of each pregnancy is held in a secure database at TAIHS, including demographic data, pregnancy characteristics, complications and outcome data, as well as cycle-ofcare data relating to clinical screening and care-planning activity. Antenatal care visits were defined as any visits to TAIHS or other services, such as Townsville Hospital or radiology visits, for documented pregnancy-related care.

Women for the PreMB group were identified from the birth register at Townsville Hospital and the TAIHS clinic register. For these women, available demographic data and antenatal visit data were collected retrospectively by TAIHS chart audit.

\section{Statistical analysis}

Relevant antenatal visit data were compared between the PreMB and MB groups, with stratification by place of residence - Townsville or "elsewhere". Depending on their distribution, numerical data were expressed as means (95\% CI), or medians (interquartile range $[\mathrm{IQR}])$. Bivariate associations were tested using $\chi^{2}$ tests, Kruskal-Wallis tests and $t$ tests, as appropriate. Cycle-of-care and careplanning data are presented as a time series for the intervention group only and examined using a $\chi^{2}$ test for trend.

Multivariate logistic regression modelling was used to identify significant predictors of inadequate antenatal care attendance (four or fewer visits). Results are presented as odds ratios with $95 \%$ CIs. The final model was adjusted for confounders. To place perinatal outcomes in context, results were compared with published state ${ }^{2}$ and national data, ${ }^{1}$ which were assumed to be the true population values.

All statistical tests were performed using SPSS, version 12.0 (SPSS Inc, Chicago, Ill, USA), or Stata, release 8 (StataCorp, College Station, Tex, USA). A two-tailed $P$ value $<0.05$ was regarded as significant.

\section{RESULTS}

\section{Study groups}

Eighty-seven women who gave birth at Townsville Hospital, 1 January 1998 - 30

\section{Demographic characteristics of women attending TAIHS for shared antenatal care, 2000-2005 (MB group) compared with a historical control, 1998-1999 (PreMB group)*}

\begin{tabular}{lcc}
$\begin{array}{l}\text { Demographic } \\
\text { characteristics }\end{array}$ & $\begin{array}{c}\text { PreMB group } \\
(n=84)\end{array}$ & $\begin{array}{c}\text { MB group } \\
(n=781)\end{array}$ \\
\hline $\begin{array}{l}\text { Mean }(95 \% \mathrm{Cl}) \text { age }- \\
\text { years }\end{array}$ & $26.0(24.8-27.2)$ & $25.3(24.9-25.7)$
\end{tabular}
years

\begin{tabular}{|c|c|c|}
\hline Aged $<20$ years & 15 (17.9\%) & $158(20.2 \%)$ \\
\hline \multicolumn{3}{|l|}{ Ethnicity ${ }^{\dagger}$} \\
\hline Aboriginal & $63(75.0 \%)$ & $511(65.4 \%)$ \\
\hline $\begin{array}{l}\text { Aboriginal and Torres } \\
\text { Strait Islander }\end{array}$ & $2(2.4 \%)$ & $53(6.8 \%)$ \\
\hline Torres Strait Islander & $19(22.6 \%)$ & $143(18.3 \%)$ \\
\hline Non-Indigenous & & $74(9.4 \%)$ \\
\hline $\begin{array}{l}\text { Marital status: single, } \\
\text { separated, or divorced }\end{array}$ & 37 (44.0\%) & $252(32.3 \%)$ \\
\hline Residents of Townsville ${ }^{\ddagger}$ & $84(100 \%)$ & $533(68.2 \%)$ \\
\hline \multicolumn{3}{|l|}{ Education level } \\
\hline Year 10 or less & na & 272/533 (51.0\%) \\
\hline Years 11, 12 & na & 168/533 (31.1\%) \\
\hline TAFE/University & na & $68 / 533(12.8 \%)$ \\
\hline Parity — median $(I Q R)^{\ddagger}$ & $2.5(1-4)$ & $1(0-3)$ \\
\hline Para 5 or higher & $13(15.5 \%)$ & $100(12.8 \%)$ \\
\hline Unwanted pregnancy & na & $73(9.3 \%)$ \\
\hline
\end{tabular}

*Data are no. (\%) unless otherwise indicated. $\dagger P<0.05$; $¥ P<0.01$ : PreMB group v MB group. Missing data: marital status (4), education level (added to the data collection in late 2001) (248). TAIHS=Townsville Aboriginal and Islanders Health Service. $\mathrm{MB}=$ Mums and Babies program. na $=$ not available. $I Q R=$ interquartile range.

June 1999, had attended TAIHS for antenatal care before the Mums and Babies program commenced. Three women with twin pregnancies were excluded from the analysis, leaving 84 women in the PreMB group.

Of the 909 women attending TAIHS for antenatal care, 1 January 2000 - 31 December 2005, 70 were excluded for the following reasons: termination (25), miscarriage/ early pregnancy loss (35), immediate referral to Townsville Hospital clinics or private obstetric care (10). The higher order pregnancies in eight women (seven twin and one triplet pregnancy) and the 50 births (6.0\%) to non-Indigenous couples were also excluded from the analysis, leaving 781 Indigenous singleton births to mothers attending the Mums and Babies program. These women made up the MB group.

The demographic characteristics of the PreMB group (historical controls) were similar to those of the intervention group, except that in the $\mathrm{MB}$ group there were significantly more non-Indigenous women $(P<0.05)$ and parity was higher $(P<0.01)$. All women in the PreMB group were residents of Townsville $(P<0.01)$ (Box 1$)$

In the $\mathrm{MB}$ group, the non-Townsville residents were significantly younger (24.3 years [95\% CI, 23.6$25.0]$ v 25.7 years [95\% CI, 25.226.1]; $P<0.01$ ), and their median parity was significantly lower than that of the Townsville residents (1 pregnancy [95\% CI, 0-2] v 2 pregnancies [95\% CI, 0-3]; $P<0.01$ ). There were no differences in any of the other demographic variables.

\section{Access}

Just under a third of the women in the MB group $(248 / 781 ; 31.8 \%)$ were not Townsville residents (predominantly from North and Far North Queensland). A total of 165 of the women in the MB group (26.8\%; 165/616) were attending for care for a second, third or fourth birth with the program.

There was a significant increase in the total number of antenatal care visits per pregnancy among the $\mathrm{MB}$ group compared with the PreMB group $(P<0.001$; Box 2$)$. Townsvillebased women made significantly more antenatal care visits $(P<0.001)$ and presented earlier $(P<0.001)$ than nonTownsville-based women. The independent risk factors for inadequate antenatal care are shown in Box 3.

\section{Quality of antenatal care}

Among the MB group, there were significant positive trends in recorded care planning $(P<0.001)$, smoking cessation advice $(P<0.001)$, and antenatal education activities $(P<0.001)$.

Pregnancy ultrasound scanning and other screening activities over time are shown in Box 4. For the MB group, the morphology scan was performed at a median of 20 weeks' gestation (IQR, 18-23) over the study period. The trends shown in Box 4 were also seen in the Townsville-based subgroup at slightly higher rates (3\%-5\% higher). Women in the MB group missing out on screening tests (either an ultrasound scan, or screening for sexually transmitted infections or a minimum blood screen) were significantly more likely to be non-Townsville residents (Townsville residents, 95/533 [17.8\%] v non-Townsville residents, 87/248 [35.1\%]; $P=0.029)$. 


\begin{tabular}{|c|c|c|c|}
\hline \multicolumn{4}{|c|}{$\begin{array}{l}2 \text { Patterns of antenatal visits for women attending TAIHS for antenatal care, } \\
2000-2005 \text { (MB group), compared with a historical control, 1998-1999 } \\
\text { (PreMB group) }\end{array}$} \\
\hline & $\begin{array}{l}\text { PreMB group } \\
\quad(n=84)\end{array}$ & $\begin{array}{l}\text { MB group } \\
(n=781)\end{array}$ & $P$ \\
\hline Antenatal visits per pregnancy - median (IQR) & $3(2-6)$ & $6(4-10)$ & $<0.001$ \\
\hline Townsville-based & $3(2-6)$ & $7(4-11)$ & $<0.001^{*}$ \\
\hline Not Townsville-based & & $5(3-7)$ & \\
\hline Gestation at first visit - median (IQR) & $14(7-22)$ & $13(8-20)$ & ns \\
\hline Townsville-based & $14(7-22)$ & $11(7-18)$ & $<0.001^{\star}$ \\
\hline Not Townsville-based & & $18(11-25)$ & \\
\hline \multicolumn{4}{|l|}{ Townsville-based women — no. (\%) } \\
\hline Pregnancies with inadequate care & $44 / 84(52.4 \%)$ & $89 / 533(16.7 \%)$ & $<0.001$ \\
\hline Pregnancies with late first visit & 15/84 (17.9\%) & $61 / 530(11.5 \%)$ & 0.004 \\
\hline
\end{tabular}

\section{Perinatal outcome}

There were improvements in all perinatal outcomes for the MB group compared with the PreMB group, but this improvement only reached significance for perinatal mortality $(P=0.014$, Box 5). Similarly, of the improved outcomes, only perinatal mortality reached statistical significance over time $(P<0.01$, Box 6). However, among Townsville-based women, there were significant improvements in perinatal outcomes in the MB group compared with the PreMB group: reductions in preterm births (from 14/83 [16.9\%] to 45/ 518 [8.7\%]; $P<0.05)$ and perinatal mortality rate (from 5/83 [60/1000 births] to $6 / 533$ [11/1000 births]; $P<0.01)$; and an increase in mean birthweight (from 3045g [95\% CI, 2861-3229g] to $3244 \mathrm{~g}$ [95\% CI, 3184$3304 \mathrm{~g}$; $P<0.05$ ), respectively.

Within the MB group, birthweight for neonates of non-Townsville-based women (3057g [95\% CI, 2929-3184g]) was significantly less than that for Townsville-based mothers (3244g [95\% CI, 3184-3304g]; $P<0.01)$. The occurrence of higher proportions of preterm and low-birthweight neonates born to non-Townsville-based mothers did not reach statistical significance (11.6\% v $8.7 \%$ and $15.5 \% \mathrm{v} 10.4 \%$, respectively).

The proportion of preterm births in the MB group (9.5\%) was similar to that for non-Indigenous births in Queensland $(8.0 \%),{ }^{2}$ and in Australia generally (7.6\%), ${ }^{1}$ but significantly lower than the proportion of preterm births to Indigenous women in Queensland in 2000-2004 (13.0\%) (Box 5) and in Australia in 2003 (14.1\%), respectively $(P<0.05){ }^{1,2}$
The mean birthweight for Indigenous Australian babies (3160 g) in 2003 was not significantly different to the mean birthweight of the MB group babies (3192 g; 95\% CI, 3136-3249 g). The proportion of lowbirthweight births in the MB group (11.7\%) was similar to the overall Indigenous rate in Queensland (12.3\%) and the national rate for Indigenous Australians (12.9\%), as was the perinatal mortality rate in the MB group.

\section{DISCUSSION}

The initial 4 years of the Mums and Babies program in Townsville improved access to quality antenatal care and significantly improved preterm birth rates, but not perinatal mortality rates. ${ }^{5}$ The program has now been running for 7 years. This article extends the evaluation to 781 births over a 6 -year period and shows previously reported gains have been sustained, and the reduction in preterm births has now translated into significantly reduced perinatal deaths.

The provision of perinatal care services that are community-based and -focused has been advocated as fundamental to the goal of improving obstetric outcomes in Indigenous communities. ${ }^{7}$ Recognising the link with chronic disease, ${ }^{8-10}$ the federal government in 2005 decided to provide significantly increased support for maternal and child health services for Indigenous communities through the Healthy for Life program. ${ }^{11}$ The Mums and Babies program in Townsville is a community-driven example of how this Healthy for Life policy initiative translates into action.

\section{Multivariate logistic regression analysis of independent predictors of inadequate antenatal care attendance*}

\begin{tabular}{ccc} 
& $\begin{array}{c}\text { Adjusted } \\
\text { odds ratio } \\
(95 \% \mathrm{Cl})\end{array}$ & $P$ \\
Predictor $^{\dagger}$ & 1.0 & $P<0.001$ \\
\hline Mums and Babies & program \\
After & $5.8(3.5-9.6)$ &
\end{tabular}

Townsville resident

$\begin{array}{ccc}\text { Yes } & 1 & P<0.001 \\ \text { No } & 2.7(1.9-3.9) & \\ \text { Parity } & & \\ \leqslant 4 \text { pregnancies } & 1 & P=0.002 \\ >4 \text { pregnancies } & 2.0(1.3-3.1) & \end{array}$

* 851 Indigenous births in Townsville, 1998-2005, in which the mother attended Townsville Aboriginal and Islanders Health Service for antenatal care, and complete data were available. (Incomplete data for smoking status, 7 women; and marital status, 7 women.)

† Smoking status of the mother was significant in the model $(P=0.036)$ before adjustment for confounding. The model was adjusted for the confounding effects of previous poor birth outcome (confounded parity), and marital status of mother (confounded parity and smoking status).

\section{Antenatal screening of all women attending TAlHS, 2000-2005, showing the increased proportion of women in the MB group screened}

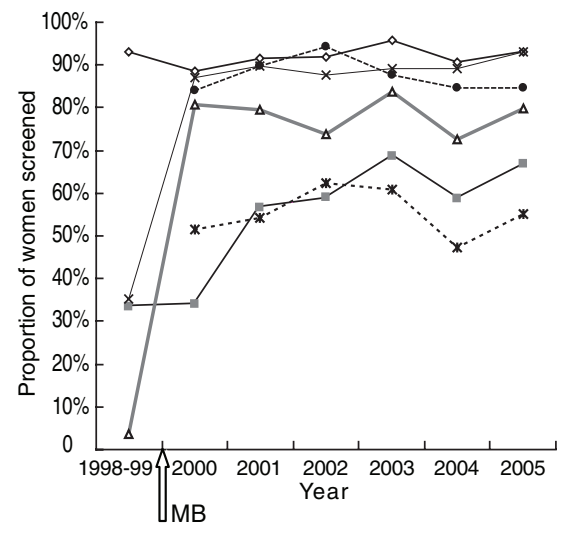

\section{$\multimap$ One or more ultrasound scans ( $95.2 \%)$ \\ - D Dating scan $(63.9 \%)^{*}$ \\ $\triangle-$ Morphology scan $(78.3 \%)^{*}$ \\ $\times$ Screening for sexually transmitted infection $(93.2 \%)^{*}$ \\ * Group B streptococcus screen (59.8\%) \\ $\ldots \rightarrow$ Haemoglobin, hepatitis B, syphilis screen (89.4\%)}

* $P<0.001$. TAlHS $=$ Townsville Aboriginal and Islanders Health Service. MB = Mums and Babies program. 


\begin{tabular}{|c|c|c|c|c|}
\hline \multicolumn{5}{|c|}{$\begin{array}{l}5 \text { Perinatal outcomes for Indigenous births to women attending TAIHS (MB } \\
\text { group), 2000-2005 compared with a historical control (PreMB group), } \\
\text { 1998-1999, and with Indigenous births in Queensland 2000-2004 }\end{array}$} \\
\hline Perinatal outcome & $\begin{array}{l}\text { PreMB group } \\
\quad(n=84)\end{array}$ & $\begin{array}{l}\text { MB group } \\
(n=781)\end{array}$ & $P$ & $\begin{array}{l}\text { QLD Indigenous } \\
\text { births }(n=14044)\end{array}$ \\
\hline Preterm ( $<37$ weeks) & 14/84 (16.7\%) & $68 / 717(9.5 \%)$ & 0.055 & $1822(13.0 \%)^{\star}$ \\
\hline Low birthweight $(<2500 \mathrm{~g})$ & $13 / 84(15.5 \%)$ & $82 / 699$ (11.7\%) & 0.289 & 1727 (12.3\%) \\
\hline $\begin{array}{l}\text { Mean birthweight } \\
(95 \% \mathrm{Cl})(\mathrm{g})\end{array}$ & $\begin{array}{c}3043 \\
(2864-3224)\end{array}$ & $\begin{array}{c}3192 \\
(3136-3249)\end{array}$ & 0.081 & - \\
\hline Perinatal deaths (PMR) & $5 / 84(60 / 1000)$ & $11 / 781(14 / 1000)$ & 0.014 & $270(19 / 1000)$ \\
\hline \multicolumn{5}{|c|}{$\begin{array}{l}\text { * } P<0.05 \text { : MB group v QLD Indigenous births, 2000-2004. } \\
\text { Missing data for gestation (64 [8.2\%]) and for birthweight (82 [10.5\%]) for births in the MB group (75\% of the } \\
\text { missing data related to births to non-Townsville residents). }\end{array}$} \\
\hline
\end{tabular}

Use of the Mums and Babies program increased rapidly from its start, and access has been maintained over the 6 years. At least $60 \%$ of Townsville-based pregnant Indigenous women are using the service, ${ }^{5}$ with about $25 \%$ returning for care of subsequent pregnancies. The timeliness of the first visit and the number of antenatal visits improved significantly. Although well short of the standard antenatal visits schedule, ${ }^{12}$ the median number of seven antenatal care visits falls within the bounds of the World Health Organization models focusing on quality rather than quantity of antenatal care. ${ }^{13}$ Residence outside Townsville was a strong predictor of inadequate antenatal attendance, as was attendance at TAIHS before the commencement of the program. The other predictor for inadequate care parity ( $>4$ pregnancies) — suggests that the team needs to further explore the barriers to attendance that these women experience. However, overall, this sustained access to antenatal care suggests that the program has successfully engaged pregnant women - a connection that should endure through the subsequent childhood years.

The proportion of preterm births in the MB group has remained lower than that for whole-of-population figures for Indigenous women in Queensland and is not dissimilar to figures for non-Indigenous women in Queensland $^{2}$ and Australia. ${ }^{1}$

The study's major limitation of selection bias - both the historical control and MB groups were self-selected - means the causative factors for these improvements in perinatal outcome remain debatable. Is it due to accurate dating of pregnancies, or differences in socioeconomic status of the Indigenous community in Townsville? The demographic profile suggests that the women in the MB group carry a significant risk load and are not of high socioeconomic status; ${ }^{14}$ and there have been no other initiatives in Townsville directed at improving pregnancy care for Indigenous women during the study period. Therefore, we believe that the improvements in perinatal outcome in the intervention group are a result of the model of antenatal care developed through the Mums and Babies program: completion of key clinical activities, together with the brief intervention for risk factors afforded by improved access to care.

Finally, the project offers potential population health gains. It generates rich, prospectively collected data that may be useful in the future and may help ameliorate the overwhelming Indigenous burden of chronic disease. ${ }^{8-10,15}$ Another significant impact is the capacity building at TAIHS, a key action in effecting health promotion, empowering people to take greater control of their health and thereby reduce health inequity. ${ }^{16}$ This has resulted in part from the framework of the program and its longevity. A quality improvement framework and continuous evaluation of this project - key components of change management ${ }^{17}-$ have played a significant role in introducing a culture of quality ${ }^{18}$ to medical service delivery at TAIHS. Evaluation has encouraged staff to engage in further education, enabled TAIHS to participate in communityfocused research projects that have added value to service delivery, enhanced primary prevention activity, and helped retain staff. The net result has been the development of sustainable health infrastructure within the organisation and a commitment to quality in health service delivery.

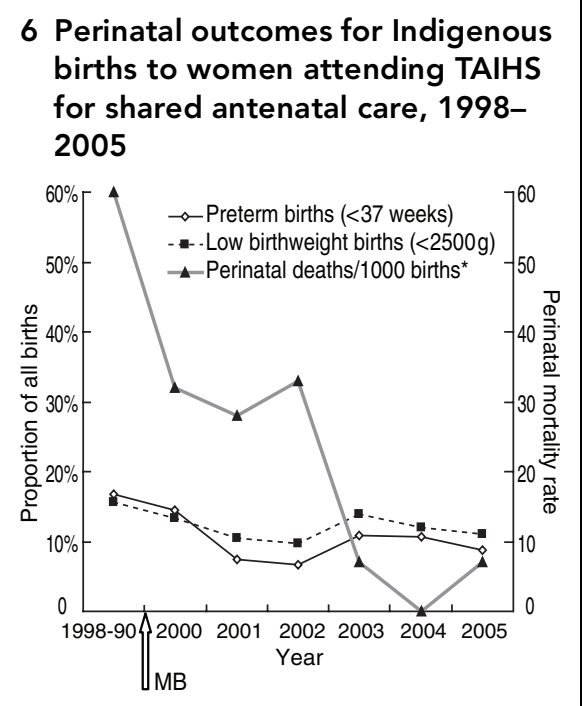

Perinatal mortality rate $=$ perinatal (stillbirths + neonatal) deaths/1000 births. ${ }^{*} P<0.01, \chi^{2}$ test for trend.

TAIHS $=$ Townsville Aboriginal and Islanders Health Service.

$\mathrm{MB}=$ Mums and Babies program.

\section{COMPETING INTERESTS}

None identified.

\section{AUTHOR DETAILS}

Kathryn S Panaretto, MB BS, MPH, FAFPHM, Medical Director ${ }^{1}$ (Currently, Population Health Medical Officer, Queensland Aboriginal and Islander Health Council, Brisbane, QLD.)

Melvina R Mitchell, Enrolled Nurse, Aboriginal Health Worker ${ }^{1}$

Lynette Anderson, Enrolled Nurse ${ }^{1}$

Sarah L Larkins, MPH, MPH\&TM, FRACGP,

General Practitioner and Senior Lecturer,

School of Medicine ${ }^{1,2}$

Vivienne Manessis, MB BS, FRACGP, General

Practitioner ${ }^{1}$

Petra G Buettner, PhD, Senior Lecturer, School of Public Health and Tropical Medicine ${ }^{2}$

David Watson, FRANZCOG, Obstetrician and Gynaecologist ${ }^{3}$

1 Townsville Aboriginal and Islanders Health

Service, Townsville, QLD.

2 James Cook University, Townsville, QLD.

3 Women's and Children's Health Institute,

Townsville Hospital, Townsville, QLD.

Correspondence:

katiepanaretto@qaihc.com.au

\section{REFERENCES}

1 Laws PJ, Sullivan EA. Australia's mothers and babies 2003 Sydney: Australian Institute of Health and Welfare National Perinatal Statistics Unit, 2005. (AlHW Catalogue No. PER 29; Perinatal Statistics Series No. 16.) http:// www.npsu.unsw.edu.au/NPSUweb.nsf/ resources/AMB_1999-2002/\$file/ps16.pdf (accessed Apr 2007). 


\section{RESEARCH}

2 Queensland Council on Obstetric and Paediatric Morbidity and Mortality. Maternal, perinatal and paediatric mortality and morbidity in Queensland, 2000-2004. Brisbane: QCOPMM, 2005.

3 Lumley J. Defining the problem: the epidemiology of preterm birth. BJOG 2003; 110 Suppl 20: 3-7.

4 Main D. The epidemiology of preterm birth. Clin Obstet Gynecol 1988; 31: 521-532.

5 Panaretto KS, Lee $H$, Mitchell M, et al. Impact of a collaborative shared antenatal care program for urban indigenous women: prospective cohort study. Med J Aust 2005; 182: 514-519.

6 Australian Bureau of Statistics. Population distribution, Indigenous Australians, 2001. Canberra: ABS, 2002. (Catalogue No. 4705.0.) http:// www.abs.gov.au/AUSSTATS/abs@.nsf/Lookup/ 4705.0Main+Features12001? OpenDocument (accessed Apr 2007).

7 O'Connor MC, Bush A. Pregnancy outcomes of Australian Aboriginals and Torres Strait Islanders. Med J Aust 1996; 164: 516-517.
8 Barker DJ, Winter PD, Osmond C, et al. Weight in infancy and death from ischaemic heart disease. Lancet 1989; 2: 577-580.

9 Barker DJ. Birthweight and hypertension. Hypertension 2006; 48: 357-358.

10 Singh G, Hoy W. The association between birthweight and current blood pressure: a crosssectional study in an Australian Aboriginal community. Med J Aust 2003; 179: 532-535.

11 Australian Government Department of Health and Ageing. Healthy for life. 2006. http:// www.health.gov.au/healthyforlife (accessed Apr 2007).

12 Hunt JM, Lumley J. Are recommendations about routine antenatal care in Australia consistent and evidence-based? Med J Aust 2002; 176: 255-259.

13 Carroli G, Villar J, Piaggio G, et al. WHO systematic review of randomised controlled trials of routine antenatal care. Lancet 2001; 357: 15651570.

14 Panaretto K, Lee $H$, Mitchell $M$, et al. Risk factors for preterm, low birth weight and small for gestational age birth in urban Aboriginal and Torres Strait Islander women in Townsville. Aust N Z J Public Health 2006; 30: 163-170.

15 Zhao Y, Guthridge S, Magnus A, Voss T. Burden of disease and injury in Aboriginal and nonAboriginal populations in the Northern Territory. Med J Aust 2004; 180: 498-502.

16 World Health Organization. Health promotion. The Bangkok Charter for health promotion in a globalized world. http://www.who.int/healthpromotion/conferences/6gchp/bangkok_charter/en/ (accessed Apr 2007).

17 Lamb MC, Cox MAA. Implementing change in the national health service. J Manag Med 1999; 13: 288-297.

18 Weber $V$, Joshi M. Effecting and leading change in health care organisations. Jt Comm J Qual Improv 2000; 26: 388-399.

(Received 8 Feb 2007, accepted 10 Apr 2007) 